\title{
Stress Induced Phononic Properties and Surface Waves in 2D Model of Auxetic Crystal
}

\author{
D. TrzupeK ${ }^{a, b, *}$, D. TWARÓG ${ }^{a}$ AND P. Zieliński ${ }^{a, c}$ \\ ${ }^{a}$ The H. Niewodniczański Institute of Nuclear Physics, PAS, Radzikowskiego 152, 31-342 Kraków, Poland \\ ${ }^{b}$ Institute of Physics, Jagiellonian University, Reymonta 4, 30-059 Kraków, Poland \\ ${ }^{c}$ Institute of Physics, Cracow Technical University, Podchorążych 1, 30-084 Kraków, Poland \\ Elastic stiffness parameters are determined in a 2D model system of rigid rods interacting by harmonic force \\ constants. Any positive ("normal" crystal) or negative (auxetic crystal) Poisson ratio can be obtained in this \\ model as a function of the external stress. Conditions for opening an absolute stop band (phononic crystal) and \\ for various kinds of surface waves are obtained.
}

PACS numbers: 63.20.D-, 68.35.Ja, 62.20.dj

\section{The model}

Auxetics, i.e. the materials with negative Poisson ratio $\sigma$, exhibit unordinary microscopic and macroscopic properties which make them useful in various biotechnological, medical and industrial applications. In particular, they serve as molecular filters and arterial prostheses. In most cases the materials are subjected to natural or man controlled external stresses transmitted by surfaces and interfaces with surrounding media.

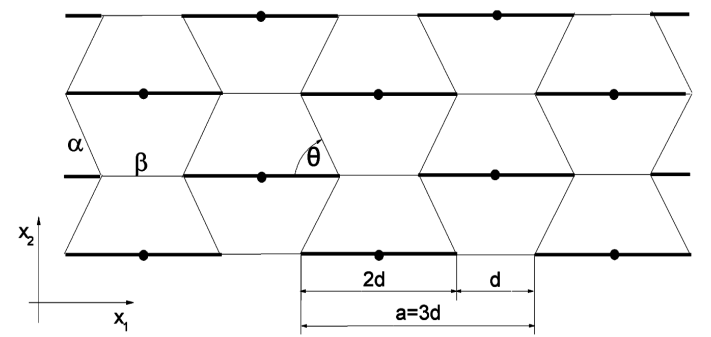

Fig. 1. Geometry of model. Rods are represented by thick lines and force constants by thin lines labeled in Greek.

The present model depicted in Fig. 1 consists of rigid rods interacting by harmonic springs and in analogy to the generic auxetic structure proposed by Evans [1] and also used e.g. in [2]. Tensile stresses $\sigma_{11}$ and/or $\sigma_{22}$ are needed to ensure the mechanical stability of the system. The force constants $\alpha$ and $\beta$ must be positive and the actual length of the spring $\beta$ must be larger than its relaxed length. The actual lengths $L_{\alpha}$ and $L_{\beta}$ of the springs differ from their relaxed values $L_{\alpha 0}$ and $L_{\beta 0}$, respectively, as a result of the applied stress. Consequently, the whole structure shows a relative elongation $\epsilon_{11}$ in the horizontal

\footnotetext{
* corresponding author; e-mail: Dominik.Trzupek@ifj.edu.pl
}

direction and $\epsilon_{22}$ in the vertical direction with respect to the generic structure with $\theta=\pi / 6$.

The free $(0,1)$ surface is modeled here by removal of the half-space $x_{2}<0$ from the original system. The masses $m_{\mathrm{s}}$ and the moments of inertia $I_{\mathrm{s}}$ of the surface rods as well as the force constants $\beta_{\mathrm{s}}$ may differ from their bulk counterparts $m, I$, and $\beta$. Also the equilibrium length $L_{\beta_{\mathrm{s}} 0}$ of the surface springs may differ from that $L_{\beta 0}$ in the bulk. At the same time we keep the actual lengths of the springs in the bulk and in the surface equal $L_{\beta}=L_{\beta_{\mathrm{s}}}$ so that perfect epitaxy is always maintained. In what follows the surface properties are studied with only tensile stress $\sigma_{11}$ applied in the $(1,0)$ direction. Therefore, the stress components $\sigma_{22}=0$.

Each rod has three degrees of freedom (two translational and one rotational) given by variables $\left(u_{1}, u_{2}, \phi\right)$. We obtain the equations of motion for the rods which in the frequency-wave vector representation take the form of the system of homogeneous linear equations for $\left(u_{1}, u_{2}, \phi\right)$. The condition of vanishing of the determinant of the corresponding dynamical matrix gives the dispersion relations for the system.

\section{Long wavelength limit}

In order to obtain the acoustic part of the lattice waves spectrum i.e. the limit of small $\omega$ and $|k| \ll 1 / d$ we expand the potential energy per unit cell in terms of the components of the gradient of displacement vector [3]:

$$
\eta_{i j}=\frac{\partial u_{i}}{\partial x_{j}}
$$

which gives

$$
E_{\mathrm{el}}=\frac{1}{2} \sum_{i j, k l} \eta_{i j} \tilde{C}_{i j k l} \eta_{k l},
$$

where the coefficients $\tilde{C}_{i j k l}$ are analogs of the elastic constants. However, because of the lack of the rotational invariance due to the external stress the coefficients $\tilde{C}_{i j k l}$ 
are related with the selected coordinate system associated with the external field and do not form a tensor. Neither they do show a symmetry with respect to interchange of indices within the first and second pair. A symmetry which holds is $\tilde{C}_{i j k l}=\tilde{C}_{k l i j}$.

When expressed by the force constants the coefficients $\tilde{C}_{i j k l}$ read

$$
\begin{aligned}
\tilde{C}_{1111} & =\frac{3\left[8 L_{\alpha 0}^{2} \beta+\alpha\left(1-3 \epsilon_{11}\right)^{2}\right]\left(1+\epsilon_{11}\right)}{4 L_{\alpha 0}^{2} \sqrt{4 L_{\alpha 0}^{2}-\left(1-3 \epsilon_{11}\right)^{2}}}, \\
\tilde{C}_{2222} & =\frac{\alpha\left[4 L_{\alpha 0}^{2}-\left(1-3 \epsilon_{11}\right)^{2}\right]^{3 / 2}}{12 L_{\alpha 0}^{2}\left(1+\epsilon_{11}\right)}
\end{aligned}
$$

The Poisson ratios in the $(1,0)$ and $(0,1)$ directions are given by $\tilde{C}_{1122} / \tilde{C}_{2222}$ and $\tilde{C}_{1122} / \tilde{C}_{1111}$, respectively, and depend not only on the constants of the model but also on applied external stress. By variating the external stress one can obtain any desired value of Poisson's ratio.

\section{Field induced phononic properties}

Dispersion relations for different external stresses are calculated along the path $\Gamma X Y \Gamma$ in the Brillouin zone (inset of Fig. 2a). The force constants are chosen to be: $\alpha=\beta=1$ and the relaxed lengths of the springs are $L_{\alpha 0}=L_{\beta 0}=d$. For higher values of external stress (corresponding to $\epsilon_{11}>0.18$ or $\theta>1.34 \mathrm{rad}$ ) the absolute stop band opens over the whole Brillouin zone. This is how the model shows a phononic behavior. Figures $2 \mathrm{~b}-4 \mathrm{~b}$ show surfaces of constant $\omega$ (slowness or inversesound-speed surfaces) in the space $\left(k_{1}, k_{2}\right)$ in the limit of small $\omega$ and $|k|$. The external stress makes the sound velocities more and more anisotropic.

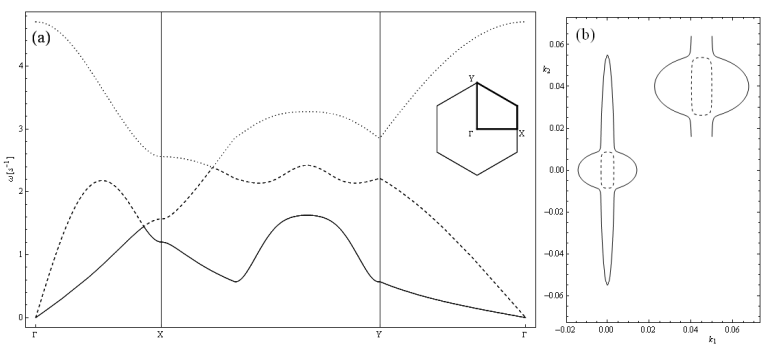

Fig. 2. Dispersion curves along path shown in inset (a) and slowness surfaces (surfaces of constant $\omega$ at small wave vector $k)$ for acoustic waves at $\epsilon_{11}=0.05(\theta=$ $1.13 \mathrm{rad})(\mathrm{b})$.
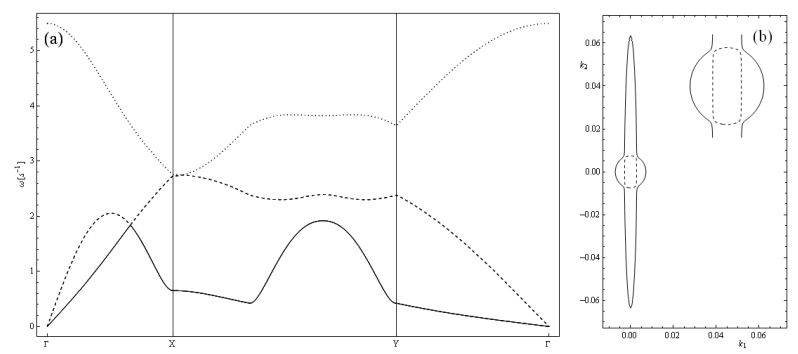

Fig. 3. The same as in Fig. 2, but with $\epsilon_{11}=0.18$ $(\theta=1.34 \mathrm{rad})$. Opening of an absolute stop band.
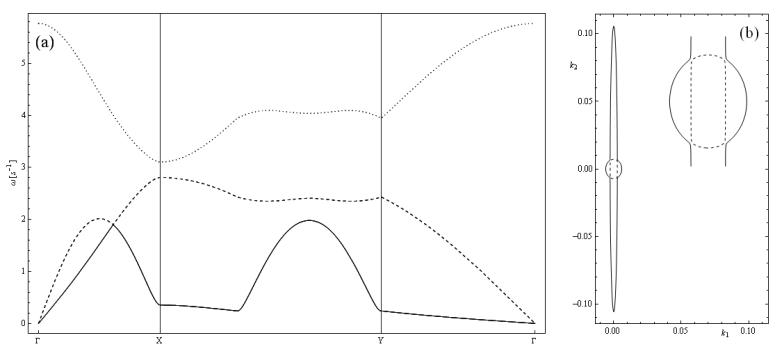

Fig. 4. The same as in Fig. 2, but with $\epsilon_{11}=0.25$ $(\theta=1.45 \mathrm{rad})$. Absolute stop band gap opened in whole Brillouin zone.

\section{Surface properties}

Figures 5 and 6 show the bulk bands and the dispersion relations of surface waves at the $(0,1)$ surface of the model. The parameters have been chosen so as to illustrate two types of acoustic behavior of the system. In Fig. 5 all the transverse acoustic waves in the long wavelength limit are slower than the longitudinal ones, whereas in Fig. 6 the transverse wave propagating in $(1,0)$ direction is faster than the longitudinal wave in the same direction which corresponds to $C_{1111}<C_{2121}$. The gray parts of the bulk bands in Fig. 5 and 6 repre- 


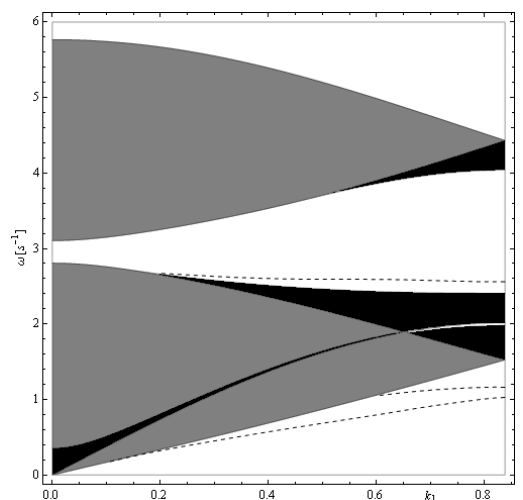

Fig. 5. Boundaries of bulk bands and surface waves dispersion curves (dashed lines) for $\beta=\alpha=1, L_{\alpha 0}=$ $L_{\beta 0}=d, \epsilon_{11}=1 / 4(\theta=1.45 \mathrm{rad}), m_{\mathrm{s}}=3 m, I_{\mathrm{s}}=3 I$.

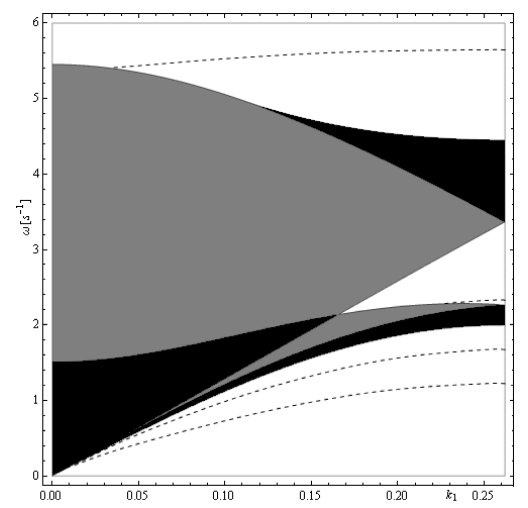

Fig. 6. Boundaries of bulk bands and surface waves dispersion curves (dashed lines) for $\beta=\alpha / 4=1, L_{\alpha 0}=$ $30 L_{\beta 0}=15 d, \epsilon_{11}=3(\theta=1.54 \mathrm{rad}), m_{\mathrm{s}}=3 m, I_{\mathrm{s}}=3 I$.

sent loci of a single bulk wave and the black parts those of two bulk waves. Generally, the condition $\sigma_{22}=0$ implies that the maximum number of bulk waves with a given component $k_{1}$ of wave vector and frequency $\omega$ never exceeds 2. Further, there exists a combination of degrees of freedom, which does not at all propagate into the material. Once excited by a perturbation of the surface this combination of degrees of freedom rests confined at the surface without forming any nearfield in the material. Any external wave exciting these degrees of freedom then is completely reflected.

\section{Conclusions}

Systems subjected to a controlable external stress may show any desired Poisson's ratio. This allows one to tune the same system from "normal" to auxetic state. Anisotropy of sound speeds as well as opening and closing of acoustic stop bands are also tunable in this way. The stress is also able to control the number and properties of surface waves.

\section{Acknowledgments}

This work was supported by National Network MANAR of the Ministry of Science and Higher Education. P.Z. acknowledges a support from the project "Ion and plasma nanotechnologies for materials formed on the basis of $\mathrm{C}$ and $\mathrm{Si}$ " in the frame of operational program Innovative Economy (Poland). The contribution of the cooperation project of the Polish Academy of Sciences and Centre National de la Recherche Scientifique (France) is acknowledged.

\section{References}

[1] K.E. Evans, M.A. Nkansah, I.J. Hutchinson, S.C. Rogers, Nature 353, 124 (1991).

[2] A. Sparavigna, Phys. Rev. B 76, 134302 (2007).

[3] J.F. Nye, Physical Properties of Crystals, Clarendon, Oxford 1957, p. 140. 